\title{
Institut für Fort- und Weiterbildung in Klinischer Verhaltenstherapie e.V. (IFKV)
}

Ausbildungsangebot

Dezember 1992 und Januar/Februar/März 1993

Ausbildung für Ausbilder

Unter dieser Rubrik wird im Januar die im Herbst 1992 begonnene «Ausbildung für Ausbilder»

fortgesetzt:

Schwerpunktthema: Was ist verhaltenstherapeutische Supervision - supervidieren lernen

Leitung: Peter Kosarz und erfahrene Supervisoren aus dem IFKV-Supervisionsteam

Termine:

9.12.1992,16.30-19.30 Uhr

Techniken und Strategien in der Gruppen- und Teamsupervision

20. 1.1993,16.30-19.30 Uhr

Techniken und Strategien in der Einzelsupervision

17.2.1993,16.30-19.30 Uhr Supervidieren lernen: Supervisionsfallarbeit

17. 3.1993,16.30-19.30 Uhr

Lernen im Dialog: Supervisoren und Supervisanden im Austausch

Die letzte Veranstaltung ist frei; alle anderen: vier Unterrichtseinheiten, Gebühr DM 100

(ermäßigt DM 80)

Ausbildung in verhaltenstherapeutischer Gruppenpsychotherapie

Beginn eines neuen Ausbildungsganges im April 1993. Anmeldung und weitere Informationen

über das IFKV-Sekretariat.

Gesamtprogramm 1993

Das Gesamtprogramm 1993 des IFKV ist erschienen und kann im Sekretariat angefordert werden. Es enthält die Angaben zu Grundkursen in Verhaltenstherapie, Ausbildung in verhaltenstherapeutischer Gruppentherapie, Ausbildung zum Verhaltenstherapeuten bei Kindern und Jugendlichen, Ausbildungsgang «Verhaltenstherapeutische Kotherapie» sowie Sonderkurse. Anmeldung und Information: IFKV-Sekretariat Kurbrunnenstraße 21a W-6702 Bad Dürkheim (BRD)

Mitteilungen der Verbände 